\title{
Alphabetic confusion: A clarification
}

\author{
RENAUD S. LeBLANC and J. GÉRARD MUISE \\ Université de Moncton, New Brunswick, Canada
}

\begin{abstract}
This paper questions the conclusion that the Luce similarity measures capture an invariant similarity structure and asserts that there is still much room for improvement in the fit of theoretical models to confusion data. Although a reanalysis of Townsend's (1971) data showed improvement in the fit of the model, the argument is made that it may be misleading to use the main diagonal elements (correct identifications) in the calculation of the correlation between two matrices as an index of fit. It is also argued that template and feature models are very weak in explaining alphabetic confusions. Because of the theoretical importance of confusions, further research is clearly warranted.
\end{abstract}

Townsend (1971a, 1971b) has presented empirical confusion matrices for uppercase letters based on group and individual data. A potentially important contribution of his work with the Luce (1963) choice model is to provide a method for isolating stimulus-similarity measures from response biases. This work has led several investigators to use the Luce similarity estimates as dependent variables (Holbrook, 1975; Stanovich, Pachella, \& Smith, 1977).

A remarkable conclusion derived from Townsend's analyses is that the choice-similarity parameters show almost no variation across experimental conditions $(r=$ $0.97)$ and across individual and group data $(r=0.99)$. This implies that there is a common underlying similarity structure that is invariant across experimental conditions and across individuals when response biases are removed. Consequently, this quasi-perfect fit of data across experimental conditions and individuals seems to leave no room for improvement. The similarity structure appears to be perfectly captured. At best, further work in the area could hope only to address the possible coherence of changes in response biases resulting from various experimental manipulations.

A second important conclusion results from Townsend's finding that a simple physical-overlap measure correlated +0.70 , or better, with the choice measures. This finding has set, for many investigators, a lower-bound estimate of $50 \%$ of the variance explained to judge the viability of alternate models (Holbrook, 1975).

This paper rejects the assumption that the Luce measures capture an invariant similarity structure and asserts that there is still much room for improvement in the fit of theoretical models to data. First, we will show that the Luce model describes confusion data even better than was suggested by Townsend. Second, we will show that, despite this improvement in fit, the cross-experimental and the interindividual correlations are far inferior to the

\footnotetext{
The authors' mailing address is: Laboratoire de Psychologie Cognitive, Département de Psychologie, Université de Moncton, Moncton, N. B., Canada ElA 3E9.
}

reported values when a different procedure is used for the calculations. Finally, we will argue that the template model is not strongly related to the choice measure, and that feature analytic models are just as weak.

\section{The Fit of the Luce Model}

Townsend used an index of fit based on the minimum sum of squared deviations between predicted and empirical confusions. Gilmore, Hersh, Caramazza, and Griffin (1979) noted a threefold decrease in the size of the sum of the squared residuals for their data relative to Townsend's. In light of this discrepancy, we decided to examine how sensitive this index is to changes in the value used as an estimate of a probability for which the proportion of observations is zero (zero probability).

Apparently, Townsend attemped fits with estimates of zero probability that varied between .000001 and .01 . However, it is not clear which specific values were tested within this range. Using the STEPIT search algorithm (Chandler, 1969), we reanalyzed Townsend's data. For each of his four published matrices, a search was made for the zero-probability estimate that would minimize the sum of the squared residuals. In addition, the fit was made to the entire matrix, to the diagonal values only, and to the off-diagonal values. Figure 1 illustrates the functional relationship among zero-probability estimates and the sum of squared residuals for the Condition 1 matrix (Townsend, 1971b). This is the matrix most commonly referred to in the literature, and the levels of improvement obtained are typical of those observed with the other matrices.

No clear rationale has yet been proposed to decide whether the goodness-of-fit measure should be calculated separately for the diagonal values and the off-diagonal values or simultaneously for both. Following Townsend's convention, we used the zero-probability estimates obtained for the entire matrix when calculating correlations between the predicted and the empirical data.

Table 1 presents the new best estimates of zero probability, the new sums of squared residuals, and the sums reported by Townsend. The table also reports the ratio of Townsend's index of fit to ours. Values of the ratio 


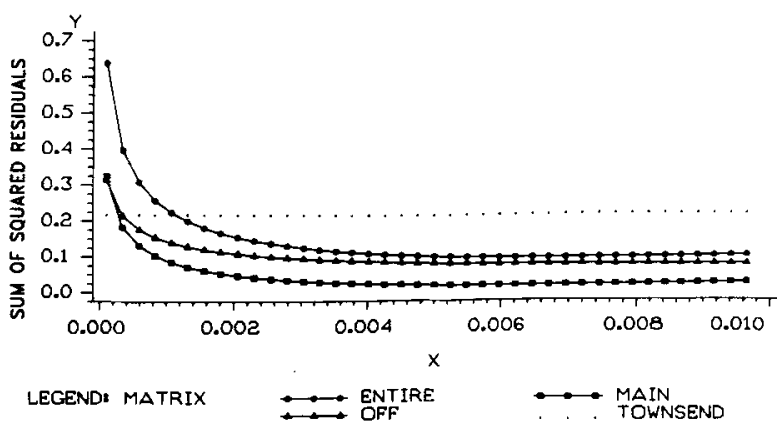

ESTIMATE OF ZERO PROBABILITY CONFUSION

Figure 1. Functional relationships between the zero-probability estimates and the sum of squared residuals.

greater than one indicate an improvement in goodness of fit for our computations. In all conditions, we obtained a better fit than that reported by Townsend. This improvement was more than threefold for the diagonal values and more than twofold for the group data. In every case, the improvement ratio was greater than one.

Our correlations were very high ( $r=0.97$ to 0.99 ) for the main diagonals (see Table 2 ). In addition, the Luce model predicts between $79 \%$ and $90 \%$ of the variance associated with the confusions. Based on this type of analysis, it is possible to confirm that the Luce model can describe alphabetic confusion data whether the diagonal or off-diagonal values are considered. On this point, we agree with Townsend.

\section{Invariant Similarity Structure}

Townsend (1971a) found correlations between subjects that are even greater $(r=0.99)$ than those found between the predicted and empirical matrices. In light of this result and of the low correlations he found for the bias parameters, he suggested that, across conditions, "bias changed and stimulus similarity did not" (Townsend, 1971b, p. 48). However, with either Townsend's zero estimates or the new estimates, it is necessary to include the main diagonals in the computations to produce coefficients in the range reported by Townsend.

A recomputation of these correlations (see Table 3), based on the off-diagonal elements only and using the new estimates of zero-probability values, provided coefficients which diverged drastically from those reported by Townsend. Because of this large discrepancy in the correlations and because of certain theoretical considerations, we disagree with Townsend concerning the inclusion of the main diagonal elements when correlating the Luce choicesimilarity measures across conditions. Consequently, we question the remarkable stability of the Luce choicesimilarity measures reported by Townsend across conditions and subjects.

Since the Luce model forces the diagonal elements to a value of 1.0 and typically produces much lower values for the off-diagonal elements, it creates a discontinuous variable, thereby artificially increasing the correlation co- efficients between matrices of similarity parameters. This discontinuity is clearly illustrated in Figure 2 ; the offdiagonal elements are clustered at the lower left and the single point at the top right represents the joint occurrence of the 26 diagonal elements. Figure 3 presents the distribution of the off-diagonal elements only.

This situation reflects the more general issue of how to compare confusion matrices. Typically, a confusion

Table 1

New Zero Estimates, Comparison of Current Sum of Squared Residuals with Townsend's, and Improvement Ratios

\begin{tabular}{|c|c|c|c|c|}
\hline & \multicolumn{2}{|c|}{ Townsend (1971b) } & \multicolumn{2}{|c|}{ Townsend (1971a) } \\
\hline & Condition 1 & Condition 2 & MJ & VF \\
\hline \multicolumn{5}{|c|}{ Entire Matrix } \\
\hline Zero estimate & 0.0070 & 0.0040 & 0.0041 & 0.0048 \\
\hline Current SS & 0.095 & 4 & 0.098 & 0.139 \\
\hline Townsend's SS & 0.23 & 0.36 & 0.18 & 0.22 \\
\hline Improvement ratio & .4 & 3.8 & 1.8 & 1.6 \\
\hline \multicolumn{5}{|c|}{ Main Diagonal } \\
\hline Zero estimate & 0.0062 & 0.0033 & 0.0048 & 0.0043 \\
\hline Current SS & 0.018 & 0.020 & 0.024 & 0.022 \\
\hline Townsend's SS & 0.07 & 0.14 & 0.09 & 0.08 \\
\hline Improvement ratio & 3.9 & 7.0 & 3.8 & 3.6 \\
\hline \multicolumn{5}{|c|}{ Off-Diagonal } \\
\hline Zero & 0.0087 & 0.0051 & 0.0029 & 0.0068 \\
\hline Current SS & 0.076 & 0.071 & 0.071 & 0.116 \\
\hline Townsend's SS & 0.16 & 0.22 & 0.09 & 0.14 \\
\hline Improvement ratio & 2.1 & 3.1 & 1.3 & 1.2 \\
\hline
\end{tabular}

Table 2

Comparison of Correlation Coefficients Between the Luce Choice Model and the Empirical Matrices

\begin{tabular}{lccccc}
\hline & \multicolumn{2}{c}{ Townsend (1971b) } & & \multicolumn{2}{c}{ Townsend (1971a) } \\
\cline { 2 - 3 } \cline { 5 - 6 } \cline { 5 - 6 } & Condition 1 & Condition 2 & & MJ & VF \\
\hline Entire matrix & 0.99 & 0.99 & & 0.99 & 0.99 \\
Main diagonal & 0.97 & 0.99 & & 0.97 & 0.98 \\
Off-diagonal & 0.92 & 0.93 & & 0.95 & 0.89 \\
\hline
\end{tabular}

Table 3

Correlation Coefficients Between the Luce Similarity Parameters Across Subjects and Conditions

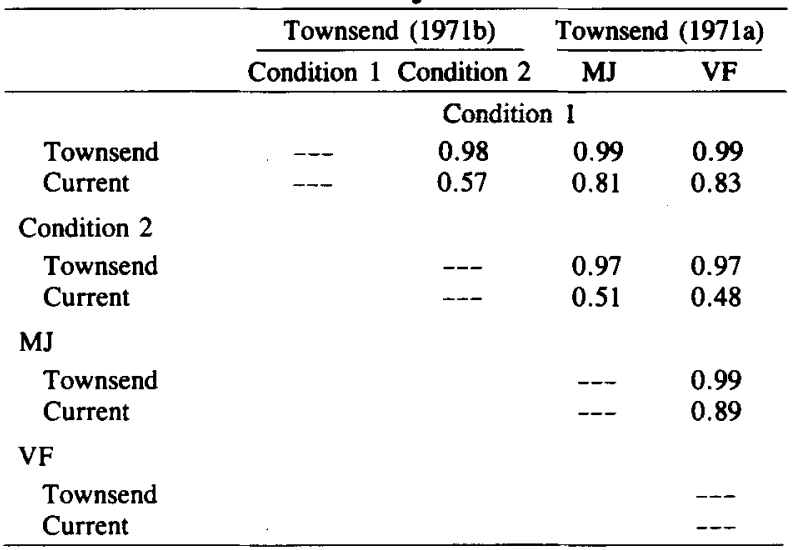




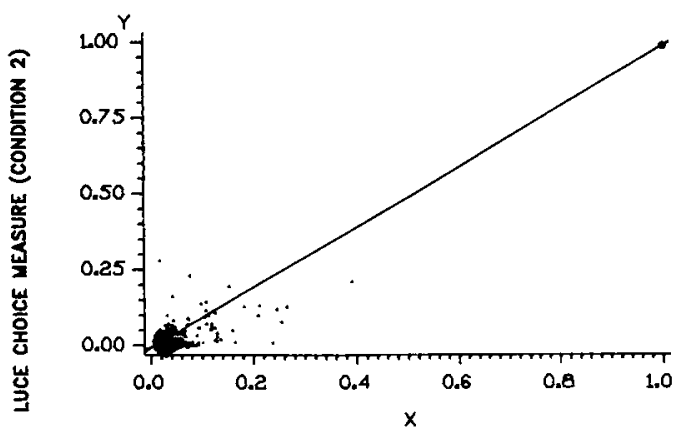

LUCE CHOICE MEASURE (CONDITION 1)

Figure 2. Scatterplot of similarity parameters including diagonal elements for Condition 1 (Townsend, 1971b).

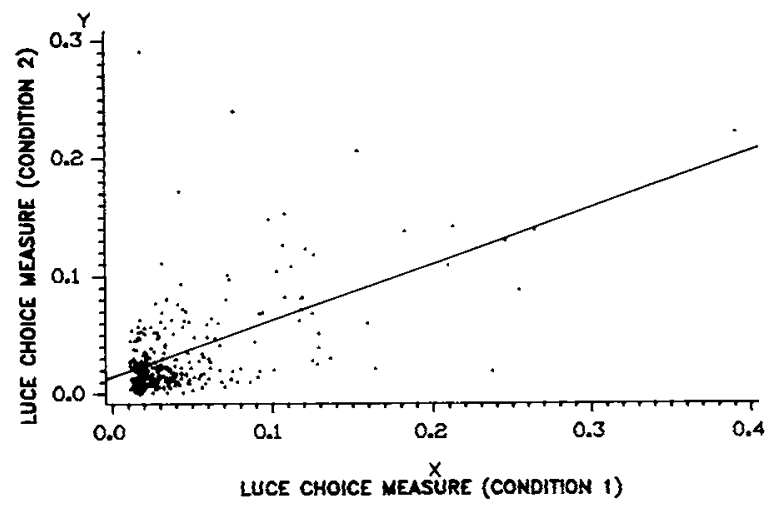

Figure 3. Scatterplot of similarity parameters excluding diagonal elements for Condition 1 (Townsend, 1971b).

matrix represents a potentially discontinuous distribution consisting of probability of correct identifications and probability of confusions. As the probability of correct identifications increases, the probability associated with confusions decreases, resulting in a significant departure from continuity. Loomis (1982) has aptly noted this aspect and considered only off-diagonal values in his comparison of confusion matrices. Because confusions directly address central theoretical issues for most theories of letter perception, it is our belief that an independent report of the goodness of fit based only on the perceptual confusions is required for any meaningful interpretation of the data (whether raw data or the sensory parameters of a model are being considered).

\section{Template and Feature Analytic Models}

The third point of this paper deals with the apparent predictive power of the simple physical-overlap model presented by Townsend. We have analyzed a number of template models using a set of computer-generated uppercase letters which are part of a general laboratory package (Muise, Theios, \& Houlihan, 1976). The correlation obtained between Townsend's simple physical model (which is based on the relative intersection of the set of points for each pair) and the choice-similarity parameters of Townsend's data is substantially lower than 0.70 . In fact, when the same template model was applied to about 20 published confusion matrices, a correlation of this magnitude was attained only once (Gilmore, Hersh, Caramazza, \& Griffin, 1979). In light of the argument presented above, the correlations between the physicaloverlap model and the Luce similarity parameters among the various conditions were computed both with and without the diagonals (see Table 4). When the diagonals are included, the correlations attain values of 0.74 (55\% of the variance explained). When the diagonals are excluded, the percentage of variance explained ranges from $2 \%$ to $22 \%$ (correlation coefficients ranging from 0.16 to 0.47 )! The substantive conclusion is that template models may not even serve as a lower bound for model comparisons.

We also question the predictive power of feature analytic models. For example, Keren and Baggen (1981) have reported using a contrast model based on the set theoretic notions of Tversky (1977). They used 29 parameters and found a correlation of 0.93 between the data and the predicted values. However, this value is based on the entire matrix and includes the main diagonal. They did not report the correlation based only on the confusions. Because of the large number of parameters used, relative to other feature models, or because of the predictive power of Keren and Baggen's model, it is possible that the correlation between the confusion data and the predicted values could be quite high.

We reanalyzed Keren and Baggen's data, using their model and the STEPIT procedure, and found a correlation of 0.95 for the entire matrix. When the goodness-offit measure was limited to the confusion data, a correlation of 0.69 was obtained (about $47 \%$ of the variance was explained). This finding is consistent with our previous arguments and constitutes a vivid demonstration of the weakness of most models when only confusion data are considered.

In summary, the Luce model is a very powerful descriptive tool and may be used with confidence to delineate the separate effects of stimulus similarities and response biases. Although quite good compared with the usual standards, the model's interexperiment and intersubject stability calculated from the perceptual confusions appears to be lower than that calculated from the entire matrix. Since the similarity parameters appear to be subject to considerable variability when only the off-diagonal values are considered, it may be possible to use them as sensitive measures of stimulus manipulation. We consequently suggest that theorists should reevaluate the predictve power of their models on the basis of the confusion data along with the usual evaluative procedures.

Table 4

Correlation Coefficients Between the Physical Overlap Model and the Luce Choice Model

\begin{tabular}{lccccc}
\hline & \multicolumn{2}{c}{ Townsend (1971b) } & & \multicolumn{2}{c}{ Townsend (1971a) } \\
\cline { 2 - 3 } \cline { 6 - 6 } & Condition 1 & Condition 2 & & MJ & VF \\
\hline Diagonal included & 0.73 & 0.61 & & 0.74 & 0.74 \\
No diagonal & 0.41 & 0.16 & & 0.47 & 0.47 \\
\hline
\end{tabular}


Finally, the use of various indices of goodness of fit renders comparisons difficult across models and experimental situations. Although Townsend (1971a, 1971b), Geyer and Dewald (1973), and Keren and Baggen (1981) all reported indices of fit which are related to the concept of "explained" variance, their various indices are not directly comparable. More specifically, Townsend used the usual sum of squared deviations between the theoretical and empirical matrices. Geyer and Dewald opted, instead, for a stress measure which is the ratio of two sums of squares: the first was the sum of squared deviations between the theoretical and empirical matrices, and the other was the sum of squared deviations between the empirical matrix and a random matrix (where each cell has a value of $1 / 26$ ). They could then report a reduction. in "total variance." Keren and Baggen also used a ratio of two sums of squares: one was the sum of squared deviations between the theoretical and empirical matrices, and the other was the total sum of squares included in the theoretical model.

We feel that it is important that workers in the field relate their preferred index of fit to the other published indices, or that they report an index which has a known distribution (Milloy, 1978) and which permits a direct comparison of models differing in number of parameters. Maximum-likelihood estimates appear to have these desirable characteristics, and Smith (1982) has shown them to be more efficient than the estimates used by Keren and Baggen (1981). This estimation method clearly warrants further exploration.

\section{REFERENCES}

ChANDLER, J. P. (1969). STEPIT: Finds local minima of a smooth function of several parameters (CPA 312). Behavioral Science, 14, 81-82.

Geyer, L. H., DEwALD, C. G. (1973). Feature lists and confusion matrices. Perception \& Psychophysics, 14, 471-482.

Gilmore, G. C., Hersh, H., Caramazza, A., Griffin, J. (1979). Multidimensional letter similarity derived from recognition errors. Perception \& Psychophysics, 25, 425-431.

HolbrooK, M. B. (1975). A comparison of methods for measuring the interletter similarity between capital letters. Perception \& Psychophysics, 17, 532-536.

KEREN, G., \& BAGGEN, S. (1981). Recognition models of alphanumeric characters. Perception \& Psychophysics, 29, 234-246.

Looms, J. M. (1982). Analysis of tactile and visual confusion matrices. Perception \& Psychophysics, 31, 41-52.

LuCE, R. D. (1963). Detection and recognition. In R. D. Luce, R. R. Bush, \& E. Galanter (Eds.), Handbook of mathematical psychology (Vol. 1), New York: Wiley.

Milloy, D. G. (1978). Comment on recognition and confusion of the lowercase alphabet. Perception \& Psychophysics, 24, 190-191.

Muise, J. G., Theios, J. M., \& Houlihan, P. J. (1976). GO8: General on-line operating system for PDP-8 series computer. Madison, WI: University of Wisconsin, Mathematical Psychology Laboratory. (DECUS No. 8-851)

Smith, J. E. K. (1982). Recognition models evaluated: A commentary on Keren and Baggen. Perception \& Psychophysics, 31, 183-189.

Stanovich, K. E., Pachella, R. G., \& Smith, J. E. K. (1977). An analysis of confusion errors in naming letters under speed stress. Perception \& Psychophysics, 21, 545-552.

Townsend, J. T. (1971a). Alphabetic confusion: A test of models for individuals. Perception \& Psychophysics, 9, 449-454.

TownSEND, J. T. (1971b). Theoretical analysis of an alphabetic confusion matrix. Perception \& Psychophysics, 9, 40-50.

Tversky, A. (1977). Features of similarity. Psychological Review, 84, 327-352.

(Manuscript received October 30, 1984;

revision accepted for publication June 3, 1985.) 